\title{
Special issue on broadband and wireless computing, communication and applications
}

\author{
Feilong Tang • Hui-Huang Hsu • Leonard Barolli
}

Published online: 9 June 2012

(C) Springer-Verlag 2012

\begin{abstract}
Ambient intelligence is an important cross-discipline research field. In order to let the background system sense humans in the environment and provide proper services to the humans, it needs sensing, networking, intelligent computing and innovative interface technologies. Various kinds of sensors can sense the movements of the human in the environment. With wired or wireless networking technologies, these sensed signals/data are then transmitted to the background computing system for analysis. Furthermore, intelligent technologies are used to understand the intention of the human from the sensed signals/data. Finally, the results are presented in the human-machine interface to interact with the human. On the other hand, the all-IP networking technology has changed the ways of living for the people around the world. The progress of electronic integration and wireless communications is going to pave the way to offer people the access to the wireless networks on the fly, based on which all electronic devices will be able to
\end{abstract}

F. Tang $(\bowtie)$

Department of Computer Science and Engineering, Shanghai Jiao Tong University, 800 Dongchuan Road,

Minhang District, Shanghai 200240, China

e-mail: feilongtang8@gmail.com

\section{H.-H. Hsu}

Department of Computer Science and Information Engineering, Tamkang University, 151 Yingzhuan Rd., Tamsui District, New Taipei City 25137, Taiwan

e-mail: huihuanghsu@gmail.com

\section{Barolli}

Department of Information and Communication Engineering, Fukuoka Institute of Technology (FIT), 3-30-1 Wajiro-Higashi, Higashi-Ku, Fukuoka 811-0295, Japan

e-mail: barolli@ fit.ac.jp exchange the information with each other whenever necessary.

The aim of this special issue is to present innovative researches, technologies and development in broadband and wireless computing, communication and applications, related to ambient intelligence. This special issue invited selected papers from the Fifth International Conference on broadband and wireless computing, communication and applications (BWCCA-2010), held on 4-6 November 2010 in Fukuoka, Japan. The authors had put a great effort to extend and enhance their work to the journal standard. All the submitted papers were strictly reviewed by three experts in the field. Totally six papers of high quality were accepted. The first two papers are related to pattern recognition and human-machine interface. The rest four are about data networking and communications.

The first paper by Lee and Li proposes fingertip handwriting alphanumeric character recognition system based on the hidden conditional random field (HCRF) model. It aims to provide an alternative way for human computer interaction. A combination of fingertip and camera provides a flexible and convenient input device. The proposed system combines fingertip detection, trajectory feature extraction, and character recognition. It also presents possible applications for camera input devices.

In the second paper, $\mathrm{Li}$ et al. use general cross-correlation (GCC) of frequency spectrum to classify various faults with fine grit. The acoustic data remotely measured by microphones are widely used to investigate monitoring and diagnose integrity of ball bearing in rotational machines. Early fault diagnosis is very difficult for acoustic emission. Principal component analysis (PCA) is used to separate the primary frequency spectrum into main frequency and residual frequency. Multi-classification strategy based on binary-tree support vector machine (SVM) is 
applied to perform faults diagnosis. The results show that this PCA-GCC-SVM method is able to diagnose various faults with high sensitivity.

In the third paper by Vitabile et al. is presented a hierarchical network architecture of several independent wireless automation cells (WAC), which are grouped in automation clusters (ACs). This work emphasizes that biometrics plays a key role in surveillance and security applications. Fingerprint, iris and voice/speech traits can be acquired by contact, contact-less, and at-a-distance sensors embedded in the environment. Biometric traits transmission and delivery is very critical and it needs real-time transmission network with guaranteed performance and QoS. The proposed architecture targets all these needs, which is evaluated in terms of authentication accuracy and network scheduling efficiency in the paper.

In the fourth paper by Oda et al. is proposed WMN-GA, which is a simulation system for wireless mesh networks (WMNs) using genetic algorithms (GAs). The authors deal with connectivity and coverage problems of WMNs. Because these problems are known to be NP-hard, the proposed system is based on GAs. The performance of the system is evaluated by different scenarios using different metrics such as client distribution, crossover rate, mutation rate, coverage area and giant component. The simulation results show that for $32 \times 32$ and $64 \times 64$ grid area, linear ranking is a good selection operator and offers the best network connectivity and user coverage.

In the fifth paper, Zhang et al. discuss policy-based mobility in heterogeneous networks. Mobility is difficult in heterogeneous networks, where heterogeneity could expand over ownership, administration, technology, and so on. There are no broadly accepted solutions for mobility in the Internet or other type of networks. The authors proposed a policy-based approach as the best and more realistic way to support mobility for heterogeneous networks. They show that by applying a policy-based approach, mobility is supported as trade-off among different aspects, depending on the respective administrations' goals. They also illustrate such capability by showing how trade-offs among security and network performance can be designed and obtained.

In the last paper Honda and Uchida give the numerical analysis of propagation characteristics of electromagnetic waves traveling along two-dimensional (2D) random rough surface (RRS). The ray searching algorithm is based on 2D discrete ray tracing method (DRTM), and the field distribution corresponding to each searched ray is computed by using source and image diffraction coefficients. Numerical computations are carried out for total field distributions along RRS as well as delay profiles at an arbitrary observation point which is far from a source antenna. Detailed discussions are made on the effect of directivity of source or receive antenna to the delay spreads of received powers.

As we conclude this overview, we would like to first thank the editor-in-chief of JAIHC, Prof. Vincenzo Loia, for his support and guidance to this special issue. We also want to thank the anonymous reviewers for giving their constructive comments to the authors, which were very helpful for the authors to further improve their papers. Finally, we wish to express our gratitude to the authors for contributing their papers to this special issue. 